\title{
EL LADO OSCURO DE LA SOCIEDAD ACTUAL: LOS RESIDUOS
}

\author{
ITHE DARK FACE OF THE PRESENT DAY SOCIETY: THE WASTE
}

\author{
Manuel Díaz Cano \\ Técnico Medio Ambiente, Santiago de Compostela. España/Spain \\ diazonac@gmail.com \\ Eduardo Díaz Cano \\ Universidad Rey Juan Carlos, Madrid. España/Spain \\ eduardo.diaz@urjc.es
}

Recibido/Received: 05/06/09

Aceptado/Accepted:03/09/09

\section{RESUMEN}

Se pretende traer a discusión la basura, los residuos, no sólo en su etapa final sino precisamente también antes de serlo, desde el origen, incluido el diseño de los productos, el consumo de éstos, la forma de deshacernos de ellos, así como el reciclaje y la reutilización por parte de los propios consumidores y empresas especializadas, técnicos, creadores y políticos. La legislación, promovida por las distintas administraciones, es otro elemento muy relevante al escuchar a los distintos actores sociales y sus demandas pero también al ir marcando la ruta a seguir para que el ser humano, como triunfador del momento, y el medio ambiente, como paciente, puedan sobrevivir a duras penas y, lo más importante, no se produzca un colapso a corto plazo porque nadie quiera soportar los desperdicios del resto o, a la larga, porque esos desperdicios tienen una vida tan prolongada que dejamos una herencia insoportable para próximas generaciones. La sociedad tiene que tomar cartas en el asunto sin tácticas dilatorias pues esto comienza a oler mal.

\section{PALABRAS CLAVE}

Ecología, legislación, consumismo, residuos, reciclaje, reutilización, basuras, incineradoras, vertederos.

\begin{abstract}
We aim to bring to discussion the waste. We try to speak about the rubbish and not only at the end of the life of the product, even before it begins, from the source, including products design, the use of these, the consumption, how to get rid of them, the citizens as well as the specialized companies, recycling and reusing them, technicians, designers and politicians. We cannot forget the legislation from the different governments. It is one of the most significant parts of this quiz. Here come together the different social actors and their proposals. It is also the way to point out the path to the human being as it is at the moment, the winner, and the environment, as the patient. Both actors are surviving with huge difficulties. It is very important that the environment does not collapse in the short term because no one wants to have in the back yard the waste of the others, or in the long run, because this waste has a so "long life" that our legacy will be unbearable for the next generations. The society has to intervene without delaying tactics; otherwise this starts soon to smell horrible.
\end{abstract}

\section{KEYWORDS}

Ecology, legislation, consumerism, waste, recycling, reuse, rubbish, waste incinerators, landfill sites. 


\section{INTRODUCCIÓN}

La sociedad tiene ante sí un gran reto, esto es, lograr que el calentamiento global no se produzca de forma exponencial. Esa lucha se hace desde diversos frentes: promoción de la vida sana, investigación (I+D), publicaciones, sensibilización sobre el consumo del agua, reducción del consumo de energías contaminantes, uso más racional -sostenible- de otros recursos naturales etc. Pero por otra parte, nos encontramos ante la espada y la pared pues si no se consume "más", la estructura social en la que económicamente nos hemos acomodado se cae, se queda casi inmóvil, la desgracia individual y colectiva se hace presente también hoy, sin esperar a la destrucción futura del entorno. Es una consecuencia de esta dinámica de sociedad que nos estamos dando, pero esta sociedad, con su compulsiva elaboración de productos protegidos, empaquetados y embolsados, para ser consumidos por los propios trabajadores, el "clímax" del que habla Bauman (2007c:19) y que debe ser alcanzado para que la maquinaria funcione ya que, de lo contrario, nos toca ser incluidos en uno de esos EREs con tan pocas expectativas a corto plazo, está creando otro problema al que no queremos enfrentarnos directamente, más bien pretendemos que permanezca lo más alejado de nosotros. Son los desechos, las basuras y su consecuencia, las plantas incineradoras o los centros de selección o valorización de los subproductos que tenemos que comprar para que la maquinaria no se pare. El problema que plantea el emplazamiento de una de estas plantas en las zonas cercanas a los lugares en los que se quiere "colocar", los diferentes grupos de población, de presión mediática y demagógica, sus líneas ideológicas y sus razones para apoyar o rechazar, por insalubres, nocivas y peligrosas, pero sobre todo por el mal olor que generan estas "industrias" será el objeto de estudio de esta comunicación. Esperamos tener un efecto contrario al del libro de Boorman (2009) No marcas. Diario de un anticonsumista, que está plagado precisamente de marcas, listas interminables que incitan más bien a su consumo; en las antípodas de nuestras pretensiones.

\section{LOS RESIDUOS EN LA LEGISLACIÓN}

Tratar sobre la normativa legislativa suele ser bastante farragoso y hablar sobre leyes en cuestión de residuos cuando intervienen cuatro administraciones con sus diferentes puntos de vista y su respectiva capacidad para legislar hace que se convierta en un encaje de bolillos el saber dónde nos encontramos aunque las competencias de cada una están claramente delimitadas además de un desarrollo y adecuación de la legislación constantes a la situación actual intentando que esas leyes sean válidas para los nuevos retos que plantea la sociedad.

La vigente Ley sobre Residuos en España, Ley 10/1998 de 21 de abril, recoge la Directiva Comunitaria 91/156/CEE de 18 de marzo. Los objetivos de la Ley, según quedan reflejados en la "Exposición de motivos" y en el "Artículo 1", son varios y van desde la adecuación de nuestra legislación a los cambios que recoge la Directiva, pasando por una mayor implicación de la legislación en la protección del medio ambiente a través de una coordinación entre las políticas de residuos, las económicas y las industriales con la finalidad de promover la reducción desde el inicio -prevenir la producción de residuos-, incentivando, por otra parte, la reutilización, el reciclado y la valorización de los residuos sobre otras técnicas de gestión. 
La Ley señala también a qué tipo de residuos es aplicable y resalta "a todo tipo de residuos" aunque señala una serie de excepciones, como por ejemplo, los residuos radiactivos o los vertidos a las aguas para los que se podrán dictar normas específicas.

Así mismo, no se olvida de las competencias en esta materia distribuidas entre el Estado, las Comunidades Autónomas y las Corporaciones locales, respetando el reparto constitucional y prevé la elaboración de planes nacionales de residuos como resultado de la integración de los respectivos planes autonómicos de gestión (el último de ellos fue publicado en el BOE $\mathrm{n}^{\circ} 49$ de 26/2/2009, Resolución del Ministerio de Medio Ambiente, y Medio Rural y Marino de 20 de enero de 2009 aprobando el Plan Nacional Integrado de Residuos para el periodo 20082015, en cuyo Apéndice II recoge todos los planes autonómicos), además, en el Artículo 3 de la Ley nos aporta una serie de definiciones.

\section{DEFINICIONES EN LA LEGISLACIÓN}

El objetivo que se persigue presentando aquí las definiciones legales es, ni más ni menos, intentar acercar la legislación al lector. Ciertamente está en el BOE, pero en pocas ocasiones acudimos directamente a él. De la basura queremos liberarnos, igual que de las leyes, pero en esta ocasión queremos que quede claro qué se debe entender cuando hablamos de:

a) «Residuo»: cualquier sustancia u objeto perteneciente a alguna de las categorías que figuran en el anejo de esta Ley, del cual su poseedor se desprenda o del que tenga la intención u obligación de desprenderse. En todo caso, tendrán esta consideración los que figuren en el Catálogo Europeo de Residuos (CER), aprobado por las Instituciones Comunitarias.

b) «Residuos urbanos o municipales»: los generados en los domicilios particulares, comercios, oficinas y servicios, así como todos aquellos que no tengan la calificación de peligrosos y que por su naturaleza o composición puedan asimilarse a los producidos en los anteriores lugares o actividades. Tendrán también la consideración de residuos urbanos los siguientes: Residuos procedentes de la limpieza de vías públicas, zonas verdes, áreas recreativas y playas. Animales domésticos muertos (animales de compañía no considerados MER, según el RD 100/2003 de 24 de enero), así como muebles, enseres y vehículos abandonados. Residuos y escombros procedentes de obras menores de construcción y reparación domiciliaria.

c) «Residuos peligrosos»: aquellos que figuren en la lista de residuos peligrosos, aprobada en el Real Decreto 952/1997, así como los recipientes y envases que los hayan contenido. Los que hayan sido calificados como peligrosos por la normativa comunitaria y los que pueda aprobar el Gobierno de conformidad con lo establecido en la normativa europea o en convenios internacionales de los que España sea parte.

d) «Prevención»: el conjunto de medidas destinadas a evitar la generación de residuos o a conseguir su reducción, o la de la cantidad de sustancias peligrosas o contaminantes presentes en ellos.

e) «Productor»: cualquier persona física o jurídica cuya actividad, excluida la derivada del consumo doméstico, produzca residuos o que efectúe operaciones de tratamiento previo, de mezcla, o de otro tipo que ocasionen un cambio de naturaleza o de composición de esos residuos. Tendrá también carácter de productor el importador de residuos o adquirente en cualquier Estado miembro de la Unión Europea.

f) «Poseedor»: el productor de los residuos o la persona física o jurídica que los tenga en su poder y que no tenga la condición de gestor de residuos. 
g) «Gestor»: la persona o entidad, pública o privada, que realice cualquiera de las operaciones que componen la gestión de los residuos, sea o no el productor de los mismos.

h) «Gestión»: la recogida, el almacenamiento, el transporte, la valorización y la eliminación de los residuos, incluida la vigilancia de estas actividades, así como la vigilancia de los lugares de depósito o vertido después de su cierre o clausura.

i) «Reutilización»: el empleo de un producto usado para el mismo fin para el que fue diseñado originariamente.

j) «Reciclado»: la transformación de los residuos, dentro de un proceso de producción, para su fin inicial o para otros fines, incluido el compostaje y la biometanización, pero no la incineración con recuperación de energía.

k) «Valorización»: todo procedimiento que permita el aprovechamiento de los recursos contenidos en los residuos sin poner en peligro la salud humana y sin utilizar métodos que puedan causar perjuicios al medio ambiente. En todo caso, estarán incluidos en este concepto los procedimientos enumerados en el anexo II-B de la Decisión de la Comisión (96/350/CE) de 24 de mayo de 1996, así como los que figuren en una lista que, en su caso, apruebe el Gobierno.

l) «Eliminación»: todo procedimiento dirigido, bien al vertido de los residuos o bien a su destrucción, total o parcial, realizado sin poner en peligro la salud humana y sin utilizar métodos que puedan causar perjuicios al medio ambiente. En todo caso, estarán incluidos en este concepto los procedimientos enumerados en el anexo II-A de la Decisión de la Comisión (96/350/CE) de 24 de mayo de 1996, así como los que figuren en una lista que, en su caso, apruebe el Gobierno.

II) «Recogida»: toda operación consistente en recoger, clasificar, agrupar o preparar residuos para su transporte.

m) «Recogida selectiva»: el sistema de recogida diferenciada de materiales orgánicos fermentables y de materiales reciclables, así como cualquier otro sistema de recogida diferenciada que permita la separación de los materiales valorizables contenidos en los residuos.

n) «Almacenamiento»: el depósito temporal de residuos, con carácter previo a su valorización o eliminación, por tiempo inferior a dos años o a seis meses si se trata de residuos peligrosos, a menos que reglamentariamente se establezcan plazos inferiores. No se incluye en este concepto el depósito temporal de residuos en las instalaciones de producción con los mismos fines y por períodos de tiempo inferiores a los señalados en el párrafo anterior.

ñ) «Estación de transferencia»: instalación en la cual se descargan y almacenan los residuos para poder posteriormente transportarlos a otro lugar para su valorización o eliminación, con o sin agrupamiento previo.

o) «Vertedero»: instalación de eliminación que se destine al depósito de residuos en la superficie o bajo tierra.

p) «Suelo contaminado»: todo aquel cuyas características físicas, químicas o biológicas han sido alteradas negativamente por la presencia de componentes de carácter peligroso de origen humano, en concentración tal que comporte un riesgo para la salud humana o el medio ambiente, de acuerdo con los criterios y estándares que se determinen por el Gobierno.

Pero como las leyes se van quedando obsoletas, ya que van unidas al momento histórico en que fueron promulgadas, también la definición de residuo, que recoge la ley 
estatal y la de distintas Comunidades Autónomas, ha vuelto a ser recogida en la Directiva 2008/98/CE del Parlamento Europeo y del Consejo de 19 de noviembre. En esta se hace una definición más precisa de diferentes conceptos -residuo, valorización y eliminación (punto (8) de los Considerandos- al establecer, dentro del término residuo, el concepto de "subproducto" (punto (22) de los Considerandos) y desarrollado en los Artículos 5 subproductos-, 6 -fin de la condición de residuo- 10 -valorización-, 11 -reutilización y reciclado- y 12 -eliminación.

En cada una de las Comunidades Autónomas, que han promulgado sus leyes, recogen esa misma definición de residuo, pero en la Ley estatal (la Directiva 2008/98/CE en el Art. 28, 4 letra c, se trata muy escuetamente de "campañas de sensibilización e información dirigidas al público en general o a un grupo concreto de consumidores") no se hace referencia a la educación, formación o concienciación de la población en general, cuestión que sí recogen tanto el Plan Nacional Integrado de Residuos, en varios apartados, como las leyes autonómicas: Galicia 10/2008, Art. 15; Cataluña 9/2008, Art. 10; Andalucía 7/2007, Art. 2, Título II en su Cap. IV; Madrid 5/2003, Art. 2, letra m, Título VIII en su Art. 64; Valencia 10/2000, Art. 19, etc. Las leyes estatales y europeas van más dirigidas a establecer mecanismos para sensibilizar, incentivar, educar, premiar o sancionar comportamientos de la administración, de las industrias, empresarios fabricantes o a los gestores de los residuos, en la línea de la responsabilidad social corporativa o empresarial, sin embargo el problema no es sólo de la generación en la producción sino de toda la cadena del consumo en la que también interviene el ciudadano de a pie.

\section{CONSUMO}

Elías (2009:20) deduce, a través de una gráfica en la que recoge 18 países, que la generación de residuos aumenta a medida que el país es más rico y, por tanto, la renta per capita disponible de los ciudadanos es más alta.

\section{Gráfico 1}

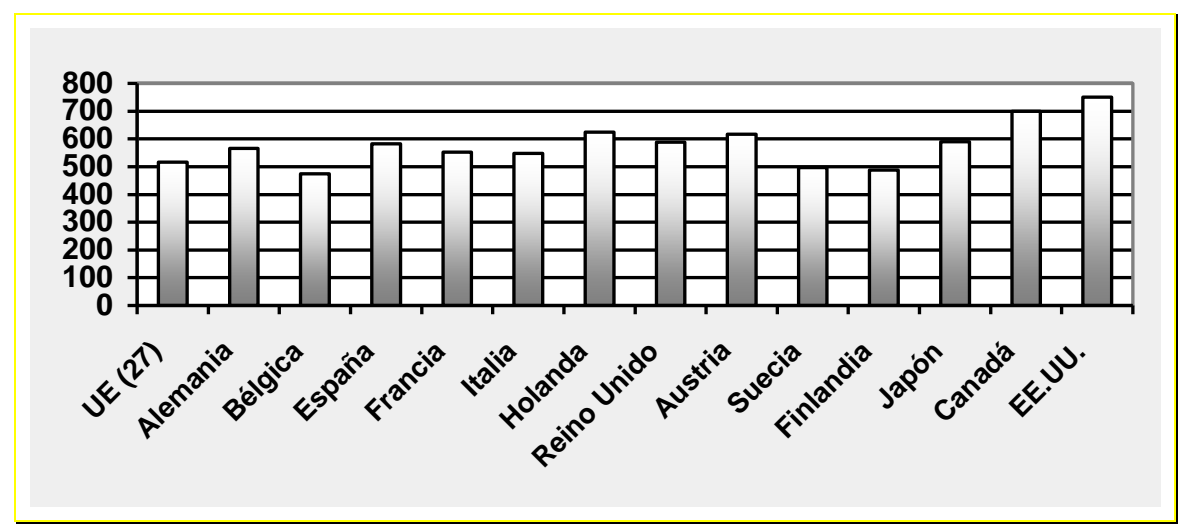

Fuente: Adaptado de Eurostat Yearbook 2008, p. 417 
Como consecuencia se observa que a igual nivel de riqueza la generación de residuos de un país es similar. En el anterior gráfico podemos ver la producción de residuos sólidos urbanos en 2008, expresada en kilos por habitante y año.

No se incluye a los dos extremos, Irlanda y Polonia, con unas cifras de 804 y 259 respectivamente, habiendo descendido en toda la UE en $5 \mathrm{~kg}$., pero con algunos contrastes: tres de los grandes países han reducido el número de kg., respecto a los datos de 2001 (Alemania, España y Reino Unido los dos primeros con casi $70 \mathrm{~kg}$.) pero otros han aumentado como Malta (110 kg.), Irlanda (100 kg.) o Dinamarca (80 kg.) quizás producido por el cambio de los procedimientos en la recogida de datos que se debe adaptar, desde 2004 al Reglamento $\mathrm{N}^{\circ}$ 2150/2002/EC que armoniza la recogida de datos para las estadísticas sobre residuos de la Unión Europea cada dos años. En España señala que Cataluña ha duplicado, en poco más de diez años, la generación de residuos per cápita.

Esta tendencia no es exclusiva de hoy, ya se refería a ello Thorsten Veblen en Teoría de la clase ociosa (2002:75) aunque el entorno es el consumo y, más concretamente, el consumo ostensible "El comienzo de una diferenciación en el consumo antecede incluso a la aparición de todo lo que pueda ser denominado propiamente fortaleza pecuniaria. Se encuentra ya en la fase inicial de la cultura depredadora y hasta hay indicios de que se encuentra una incipiente diferenciación en este sentido antes de los comienzos de la vida depredadora. ... La utilidad del consumo como demostración de riqueza ha de clasificarse como proceso derivado. Es una adaptación" Pero esta adaptación, como dice Alonso (2005:129) no es exclusiva de las primeras fases de la cultura depredadora, se repite a lo largo de la historia y está en alza desde finales de los años ochenta, los yuppies o brokers han consolidado en su entorno un nuevo segmento de consumo ostentoso. Consumo que tiene como consecuencia una desorbitada utilización de recursos, que no necesariamente podremos seguir teniendo a disposición durante mucho tiempo. Evidentemente el consumo ostentoso, del que habla Veblen no es el mismo del que podemos hablar ahora, Lipovetsky (2007:120141) utiliza el término homo consumericus, el "consumo-mundo" o más aún, el "consumomundo tentacular", o el "turboconsumidor" y, si en su época los recursos eran tan escasos como ahora, la posibilidad de disfrutar de ellos estaba limitada a un número de consumidores reducido. La situación actual, por el contrario, ha cambiado radicalmente por el simple hecho de que de forma masiva se quiera -o pueda- tener acceso a esa forma de consumir, no tanto por necesidad, más bien por ostentación. Pero qué entendemos por "recurso": Recurso es todo material que precisa un organismo, una población o ecosistema en cantidad suficiente para permitir elevar su tasa de conversión de energía. Es decir, el recurso natural es tanto la materia como la energía presentes en el medio natural utilizable por el hombre y ese medio natural se convierte, en tanto que es utilizado por el hombre, en su medio ambiente al que, a su vez, podemos definir como el conjunto de sistemas físicos y biológicos que aparecen como resultado de la interacción del hombre con el hábitat que le rodea $\mathrm{y}$, esa interacción ¿tiene límites? ¿Podemos disponer de recursos naturales de forma ilimitada?

Evidentemente, las respuestas a estas preguntas son: sí existen límites, especialmente para aquellos recursos que no son renovables e incluso para los renovables, como por ejemplo la energía solar, tiene el límite temporal que marca la salida y la puesta del sol, igual sucede con el viento $u$ otros recursos naturales sobre los cuales todavía no disponemos de la tecnología suficiente como para "almacenarla" en tal cantidad que se pueda utilizar en el momento en que sea necesario y con la misma intensidad como se hace actualmente con otras fuentes de energía no renovables, como por ejemplo el petróleo o sus derivados, más aun, si el crecimiento de la demanda, tanto para usos generales como 
ostentosos -entiéndase aquí "ostentoso" en el sentido de superfluo y no imprescindible, rayando en el despilfarro- sigue aumentando como en los últimos años en todos los niveles sociales y en los cuatro puntos cardinales, ¿podrá seguir el medio ambiente, el recurso natural, soportando tal aumento de la demanda? ¿Podemos hacer depender de la tecnología la respuesta a esta pregunta? Posiblemente la tecnología -los científicos- nos sigan sorprendiendo hasta límites insospechados, pero con la tecnología actual no es posible mantener el mismo nivel de consumo que lleva la sociedad en la que vivimos. Analicemos simplemente las consecuencias que nuestro actuar tiene sobre el medio ambiente y la sobre explotación a la que sometemos a los recursos naturales, por otra parte vemos, con esperanza, que la tecnología avanza hacia un mejor aprovechamiento de los recursos, pero sólo cuando nos hemos dado cuenta que estos son limitados o cuando los beneficios económicos de los diferentes proyectos han podido ser meridianamente constatados.

El Informe del Club de Roma en 1972, el Informe Meadows, afirmaba que "Si las tendencias actuales en cuanto a población, industrialización, contaminación, producción de alimentos y agotamiento de recursos no se modifica, se alcanzarán los límites del crecimiento en 100 años" y si bien es cierto que el consumo ha seguido aumentando desde entonces iy a qué ritmo! También es cierto que no sabemos cómo solucionar algunos problemas que afectan a la supervivencia del ser humano como tal y me refiero a los efectos del calentamiento global (los científicos de la ONU ya datan el fin del hielo en los polos para 2030!), difíciles de evaluar por no tener una respuesta inmediata, un feedback instantáneo para poder poner remedio instantes después de habernos dado cuenta que nuestro actuar está causando un mal irreparable a corto plazo a nuestro medio ambiente, efecto que puede causar la nueva tecnología -o no- ya que el proceso de creación y la urgencia con que se quieren implantar determinadas tecnologías no garantizan que esa tecnología sea beneficiosa para el ser humano y, por lo tanto, tampoco para el medio ambiente.

Parece claro que se podría llevar a cabo un mejor aprovechamiento de los recursos naturales pero, pensando que el crecimiento de la población mundial no se va a estancar y que los niveles de bienestar deseables tampoco van a ser cuestionados y van a mantenerse en la esfera de lo individual, del ser soberano que es cada individuo. Pero hoy, unos años después del Fordismo, de la Pirámide de Maslow, del Estado del Bienestar y de la Caída del Muro de Berlín, disfrutamos de unas cadenas de montaje totalmente automatizadas en las que ni Charles Chaplin tendría espacio, una carrera por ascender en la Pirámide tan rápida como la de Usain Bolt y que no es posible mantener a todas luces pero que el Estado del Bienestar facilita -casi facilitaba- así como el Estado, al otro lado del Muro de Berlín, lo intentaba de una forma racional-científica y para todos igual pero que tampoco lo lograba de forma inocua, el medio ambiente sufría las mismas consecuencias.

Pero esta teoría individualista, en la que se pone de manifiesto el axioma de "el cliente siempre tiene razón" pasando a considerar que el consumidor es el rey, ha pasado a un segundo plano. Ciertamente el consumidor tiene que seguir yendo a las tiendas o conectarse a Internet para entrar en una tienda virtual o una subasta para hacerse con alguno de los productos más novedosos, económicos o cualquier otra característica que queramos atribuirle a la nueva "supertienda" que es Internet pero en el fondo representa la misma tendencia que la de las grandes compañías, multinacionales, corporaciones o empresas globales, son estas empresas las que van marcando, educando, al consumidor para que se acerque a su local pues siempre ofrecemos lo mejor, lo más novedoso, lo más barato, los más rebajado, etc., todo estrategias de venta para hacerse con ese nicho de mercado y en el que, naturalmente, juega un papel fundamental la mercadotecnia. 
Nos puede surgir la duda ¿es el individuo o la empresa quién decide sobre qué consumir en cada momento? Evidentemente es el individuo pero para ello la empresa ha comprado a todos sus competidores en los alrededores, ha bombardeado al individuo con cientos de anuncios en los medios de comunicación masivos y elitistas, han puesto sus productos en todas las tiendas, incluso en las de la competencia o, para rizar el rizo, se ha puesto de acuerdo con su competidor para que sus empresas no compitan entre sí sino que compitan por el consumidor en la línea del supermercado, repartiéndose, de esta forma, el mercado y poniendo difícil la llegada a la tienda, tanto a la física como la supertienda, de nuevos competidores. Es la empresa la que va formando el mercado al que entra el consumidor que es quien, en definitiva, da continuidad a esa propuesta empresarial y él, en su individualidad, se va acostumbrando a unas prácticas, iguales pero diferentes, de ahí la capacidad de elección según el gusto, marcado por las propiedades relacionales, es el habitus de Bourdieu (2003) y para que esos consumidores tengan a su disposición los productos ofertados, deben ser fabricados por esos mismos consumidores, son los prosumidores de Toffler (1981) y llevado al entorno Internet -Second Life y otros- por Tapscott y Williams (2007:189 y ss.) pues si esos productores-consumidores no consumen son ellos mismos los que se destruyen su castillo de naipes, los que hacen descender el volumen de negocio y, de esa forma, su bienestar, en el caso de Internet a que desaparezca la realidad-comunidad “virtual", creada, producida en un $99 \%$ por los mismos consumidores-jugadores. Ahora bien, ¿debemos plantearnos el problema desde ese punto de vista? o debería ser en sentido contrario: la cuestión es el bienestar, no el volumen de negocio, como nos propone Weizsäcker (1997:358-362) pero reconoce que, en épocas de crisis y por lo tanto extrapolable a la actual, el volumen de negocio es imprescindible que aumente, en caso contrario aumenta el volumen de parados. La aportación de Weizsäcker es la búsqueda de alternativas para que no se sigan separando tan rápidamente como hasta antes de la crisis ambas líneas, la de volumen de negocio y la de bienestar.

Para Verdú (2005), sin embargo, este consumismo, este individualismo -narcisismoesta energía del placer de una época que toca a su fin, son la muestra de un nuevo sobjeto que actúa en la búsqueda de una felicidad especialmente relacionada con los múltiples nexos con los demás, por superficiales y efímeros que sean los contactos, es el buscar el trato con los demás como sobjetos, sujetos y objetos a la vez, nuevos objetos de lujo. Es Zara, Dell o Harry Potter, es comprar, como elección personal, pero también como el rito de formar parte del acontecimiento en sí, la moda o la novela, en caso contrario estaría fuera de esa corriente a la que pertenece, corriente global, interpersonal, suprapersonal, planetario, que no son duraderas, sólo ocurrencias. El consumismo es, así, oportunismo, la prenda excelente hallada por azar en las rebajas: es el personismo. (Verdú, 2005:36 y 197). "Si uno no quiere hundirse, debe seguir haciendo surf', dice Bauman al respecto (2007a:146) y continua "y eso implica cambiar de vestuario, de muebles, de papel pintado, de aspecto y de hábitos: No necesito añadir, por obvio, que este nuevo énfasis en deshacerse de las cosas, en quitárselas de encima y desprenderse de ellas, en vez de apropiarse de ellas, es algo que obedece a la lógica de la economía orientada al consumo. Que la gente conserve la ropa de ayer, así como el ordenador, el teléfono móvil o los cosméticos podría significar el desastre para una economía cuyo interés, y también la condición sine qua non de su supervivencia, es que los productos vendidos y comprados vayan a la basura con rapidez y sin dilación; y en este aspecto de la economía el saber qué hacer con lo que sirve es en sí una industria de máxima calidad".

Pero no todo es blanco sobre negro, Lipovetsky afirma casi lo contrario, en una primera parte, cuando sugiere que Narciso no está tan enamorado de sí mismo como 
aterrorizado por la vida cotidiana, por su cuerpo y por su entorno social que se le antoja agresivo, coincidiendo con él en que lo que caracteriza a este neoindividualismo es el rechazo prometeico del destino y la invención de uno mismo sin vía social trazada de antemano (Lipovetsky, 2003:27). Es, en esa primera parte, donde tiene espacio el consumismo que funciona, desde ahora, como doping o como animación de la existencia, y a veces como paliativo, como diversión para todo lo que no funciona en nuestra vida.

Y si damos por aceptable esta afirmación nos damos cuenta que es uno de los cientos de resquicios que aprovechan las empresas para tratar de colocar sus productos, productos de todo tipo y para todo tipo de consumidores. No hay posibilidad de escaparse. Naturalmente que las empresas producen de forma y manera ética, pero el mismo Lipovetsky no volvería a recoger en su discurso los "fondos éticos" de los bancos que enumera en el texto relativo a la empresa (2003:61-96). En la empresa, la ética puede funcionar como objeto de reclamo, pero está comprobado que no aporta casi nada en volumen de ventas pero sí ejerce una muy negativa influencia si se descubre que no se trabaja con ética en los sistemas de producción, en cualquiera de sus ámbitos. Por lo que este podría ser el próximo campo de análisis para este trabajo. ¿Por qué siguen fabricando productos cuyos creadores, casi con toda seguridad, son conscientes de que no son inocuos ni para las personas ni para el medio ambiente? En la tabla 1 aparecen algunos de los productos "clásicos" recuperados.

Tabla 1

Millones de habitantes en $2007 \mathrm{y}$

recuperación de residuos, valores de 2004 y en 1.000 Tn.

\begin{tabular}{|l|r|r|r|r|r|r|r|r|}
\hline & Habit. & Metal & Vidrio & Papel & Goma & Plástico & Madera & Textil \\
\hline Alemania & 82,3 & 6.990 & 2.162 & 6.054 & 179 & 518 & 2.221 & 109 \\
\hline Francia & 63,4 & 17.210 & 2.000 & 7.550 & 268 & 380 & 4.261 & 302 \\
\hline R. Unido & 60,8 & 4.397 & 1.931 & 6.891 & 142 & 1.479 & 2.715 & 284 \\
\hline Italia & 59,1 & 6.981 & 1.429 & 3.335 & 49 & 959 & 4.248 & 244 \\
\hline España & 44,5 & 4.953 & 1.335 & 2.217 & 332 & 2.646 & 279 & 127 \\
\hline Polonia & 38,1 & 6.446 & 489 & 1.157 & 68 & 279 & 930 & 111 \\
\hline Rumanía & 21,6 & 4.077 & 76 & 344 & 2 & 12 & 80 & 4 \\
\hline Holanda & 16,4 & 1.344 & 453 & 2.667 & 71 & 251 & 1.209 & 78 \\
\hline Grecia & 11,2 & 2.324 & 64 & 263 & 3 & 4 & 24 & 4 \\
\hline Hungría & 10,1 & 577 & 18 & 287 & 23 & 23 & 183 & 2 \\
\hline Austria & 8,3 & 1.615 & 251 & 1.156 & 21 & 349 & 2.935 & 120 \\
\hline Dinamarca & 5,4 & 606 & 140 & 677 & 76 & 54 & 721 & 0 \\
\hline Eslovaquia & 5,4 & 910 & 19 & 45 & 9 & 24 & 126 & 10 \\
\hline Irlanda & 4,3 & 11 & 3 & 118 & 0 & 10 & 155 & 11 \\
\hline
\end{tabular}

Fuente: Adaptación de Eurostat Yearbook 2008, habitantes p. 25; residuos p. 419.

La razón puede ser doble: no existen otras "materias primas" más inocuas para esos productos demandados por el público o la empresa considera en segundo lugar las consecuencias y en primer lugar los beneficios. En palabras de Galbraith "La economía y los 
grandes sistemas económicos y políticos cultivan su propia versión de la verdad de acuerdo con las presiones pecuniarias y las modas políticas de la época" (Galbraith, 2004:11) y esto deja al descubierto que esa versión no tenga necesariamente relación con lo que ocurre en realidad.

Somos conscientes de varios ejemplos recientes: los gases efecto invernadero (CFC), el plomo de las gasolinas, el tabaco y las bolsas de plástico. Tres de estos productos se introdujeron en la industria para obtener mejoras en la calidad de vida de los consumidores, pero no se conocían todos los efectos nocivos que su uso iba a ocasionar y, si en un principio, cuando su uso estaba limitado a un número de población relativamente pequeño, al pasar a uso masivo, las consecuencias fueron y siguen siendo muy negativas, de tal manera que la misma sociedad se ha llegado a poner de acuerdo para restringir o eliminar su uso. El tabaco es otra cuestión, ya que, y sin que sirva de alegato a favor del consumo de esta planta, uno de sus problemas radica en los muchos aditivos que se introdujeron con una finalidad muy diferente del simple disfrute, del deleite de una hoja al inhalar su humo relajante y narcotizante, como lo siguen haciendo en múltiples lugares de forma natural, como lo hacían sus ancestros. Sin embargo, y enlazando con otro de los hilos conductores del texto, algunos locales (léase bares, tascas, pubs, restaurantes, etc.) protestan ante quienes quieran escucharles por la nueva reglamentación que se quiere introducir para restringir al máximo el humo procedente del tabaco en los lugares públicos, es decir, en los bares, como aquellas islas en las que se podía autorizar el consumo del tabaco sin ser penalizados por ello, pero hoy, en el siglo XXI y sabiendo los aspectos negativos que puede acarrear esta práctica, los empresarios, algunos de ellos, demandan de la administración que siga permitiendo el fumar en sus locales "porque de lo contrario se irían a la ruina, en una situación de crisis como la actual" o en términos semejantes. Es decir, la salud importa poco, las arcas públicas casi nada y la contaminación menos, lo que importa es el negocio, aunque venga de la mano del tabaco.

La campaña de reducción de las bolsas de plástico tiene sus aspectos beneficiosos para el medio ambiente pero el problema se trata de solucionar sólo a medias pues, seguramente, desaparecerán de las cajas de los supermercados, pero aun no se ha dicho nada de las que utilizamos para la basura -y que reutilizábamos de la compra- y a partir de ahora tendremos que comprar un volumen semejante y en exclusiva para que sirva de envoltorio de los cientos de productos que nos llevamos a casa y desechamos por inútiles. Si el problema es el plástico y lo es por sus residuos, la solución debe de ser completa y las empresas fabricantes deberían ponerse manos a la obra para encontrar otras propuestas que realmente le den solución sin crear otro mayor, como ya defendíamos en otro texto anterior al recordar la frase de Einstein "El hombre y su seguridad deben constituir la preocupación fundamental de toda aventura tecnológica" y añadimos "científica”. (Díaz Cano, 2008:259).

\section{LOS RESIDUOS}

El recordatorio anterior es efectivo siempre y cuando el beneficio económico no sea lo principal y sí la sostenibilidad de la empresa y sus productos. Imaginemos, por un momento, a todo el mundo consumiendo la misma cantidad de materia y energía. ¿Durante cuánto tiempo podríamos mantenerlo? Casas, coches, viajes, productos naturales procedentes de cualquier parte del mundo y a cualquier hora. El modelo de fordismo global, indica Altvater (1994:163) es excluyente, es decir, excluye a una gran parte de los seres humanos de 
las gratificaciones del bienestar, pero ¿cómo regular la contradicción entre desarrollo y medio ambiente? Pues el aprovechamiento de ciertos recursos amenaza la capacidad de regeneración ecológica de ecosistemas locales, regionales y globales. Los máximos costes globales del fordismo son las emisiones de sustancias tóxicas a la atmósfera y de residuos en general. Las sufrimos todos los seres humanos, tanto en el norte como en el sur, desarrollados o en proceso y deseos de desarrollarse. Es una externalización de los costes de producción de riqueza.

¿Cómo se enfrenta el individuo, causante de la contaminación y sensibilizado con el problema medioambiental a la contradicción de seguir manteniendo su régimen de consumo y de generación de residuos?

Podemos resaltar dos: los que no hacen casi nada porque ven el problema como algo lejano y contra lo que su acción individualizada no surte efecto a corto plazo, y los que se hacen responsables de su pequeña parcela e intentan aportar su granito de arena para mejorar aquello que, individual o colectivamente, se considera que es erróneo o dañino para el medio ambiente y comienzan a apagar las luces, cuando no se necesitan, a utilizar algo más el transporte público, a viajar más despacio con el vehículo privado, a no despilfarrar energía con el uso de la calefacción/aire acondicionado en invierno y verano, etc.

Igual sucede con las empresas, quienes, sorpresa si lo decimos en este punto del texto, ya en 1989 apareció el concepto de cleaner production, propuesto por el PNUMA (Programa de las Naciones Unidas para el Medio Ambiente), es decir la producción más limpia que se define como la forma de producción que considera todas las fases del ciclo de vida de un producto o de un proceso con el objetivo de prevenir o minimizar el riesgo para los humanos y el medio ambiente a corto plazo y que desde 1994 queda definido como la aplicación continua de una estrategia ambiental preventiva integrada en procesos, productos y servicios a fin de aumentar la ecoeficiencia y reducir el riesgo para los humanos y el medio ambiente, lo que viene a estar representado por la rueda de Deming:

Planear: Documentar el proceso, analizar datos, selección del procedimiento, establecer metas cuantitativas, elaborar el plan de mejora;

Ejecutar: Aplicar dicho plan de mejora, observar los progresos, documentar cambios;

Comprobar: Analizar los nuevos datos, observar las desviaciones respecto a los objetivos, detectar las limitaciones;

Actuar: Mejora de las debilidades, afianzar las fortalezas y definir las nuevas mejoras.

Pero esto está vigente desde la década de los noventa, es la prevención basada en los sistemas de gestión ambiental (SGA) y, aunque significaron un avance por normalizar la gestión medioambiental de las empresas, concretado en EMAS (Eco Management and Audit Schema) y en la ISO 14001, queda mucho por andar, dado que los efectos son aún poco visibles o las expectativas están puestas muy alto.

No quisiéramos quedarnos por debajo de las propuestas de Braungart y McDonough (2005:99): diseñar productos que, una vez utilizados, puedan volver a los ciclos naturales. Realidad difícil de conseguir, pero debemos marcarnos metas y tratar de lograrlas.

Pero aun eso, tenemos el problema presente al que resulta difícil dar solución. Son los residuos ¿Qué hacer con aquellos residuos que no pueden volver al ciclo natural?

Por ejemplo, en el Gráfico 2 no aparece ni Gran Bretaña ni Francia, con tasas de implantación mínimas. 


\section{Gráfico 2}

$\mathrm{N}^{\mathrm{o}}$ de instalaciones que han incorporado el EMAS o el ISO 14001, 2006

(Por millón de habitantes)

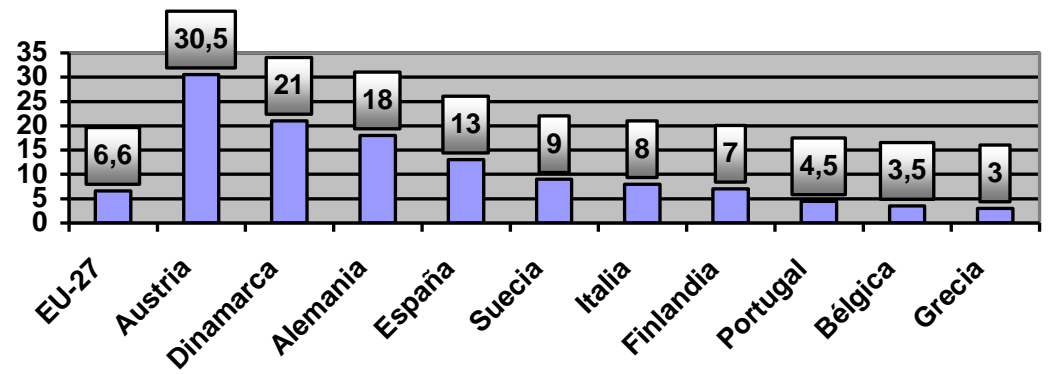

Fuente: Adaptado de Eurostat Yearbook 2008, p. 430

Está claro que el primer paso es retirarlos de la vista de sus generadores (nosotros) si bien en ocasiones son utilizados como transacción política y se amontonan durante días en las calles como suele suceder en verano en algunas de nuestras ciudades más turísticas o, hace unos meses, en las ciudades italianas de Nápoles y Palermo, siendo en Nápoles, y su región La Campania, donde se encuentra el vertedero más grande de Europa, a donde llegaban basuras no sólo de la ciudad y de los alrededores, también del resto de Italia e incluso de toda Europa y si los habitantes napolitanos no padecieron el Síndrome NIMBY (Not in my back yard) hasta principios de los 90 fue porque se hizo casi de forma clandestina, desde entonces no son receptivos -sus lagunas, ríos y campos agrícolas están altamente contaminados y el índice de cáncer en la región ha aumentado un $20 \%$ en los últimos añosahora ya no son receptivos a los desmanes de los demás ciudadanos, de las empresas, de los políticos, de los funcionarios corruptos y de la misma Camorra, quienes sí ven a la basura como una fuente de oro líquido, pero que no ha sido gestionada de forma eficaz y simplemente se ha amontonado en los distintos vertederos tanto legales como ilegales. Hasta que se encuentre una solución definitiva al problema, se enviará, como se ha estado enviando, la basura a incineradoras alemanas y suizas, de forma excepcional y por una cantidad limitada incluso a la isla de Cerdeña, lugar de destino de la jet-set europea, aunque según datos de los tres autores citados, el envío se realiza desde hace siete años y hasta el 2007 se seguían recogiendo residuos peligrosos en basureros ilegales procedentes del norte y centro de Italia (Dean, 2008; Mora, 2008 y Lucchini, 2008).

Vertidos ilegales, incendios de basuras, traslados no autorizados, son la consecuencia de una mala planificación o de una falta de previsión de nuestros políticos y técnicos responsables. En España disponemos de una legislación lo suficientemente clara y al día como para que no se produzcan desmanes como el que hemos enumerado anteriormente, pero la realidad no siempre atiende a lo que dicta la legislación pues está en boca de todos que es más barato contaminar que aplicar las medidas correctoras. Queremos pensar que esto está quedándose obsoleto y que los planes municipales, comarcales, provinciales, autonómicos, nacionales e internacionales lo tienen todo pensado para que nada se colapse y todo funcione dentro de unos límites saludables. 


\section{DUDAS}

Al comienzo hablábamos de las distintas legislaciones y como éstas se van adecuando a las necesidades actuales, pero no todo es de color rosa. El último caso del que se han hecho públicos algunos datos (pero no exclusivo pues también aparecen en las noticias el Principado (La Zoreda), Cantabria (El Mazo), Cartagena (El Gorguel y Mina Regente), Murcia (Cañada Hermosa), Sagunto (Pic dels Corbs), Castellón (Vilafranca), Palencia (Cevico de la Torre), Lanzarote (Zonzamas), etc.) es el de Sogama que, según el artículo, "dispone de una planta completamente colapsada en Cerceda, y teniendo que recurrir a enterrar sin ningún tipo de tratamiento la mitad de los desechos que recibe" (Nogueira, 2009) y enunciemos aquí solamente, por tratarse de un despropósito difícil de entender, el caso que retorna una y otra vez a los medios locales de comunicación (llevan 9 años esperando una solución) sobre las "balas de basura" -miles de toneladas de residuos empaquetados en bolsas de basura y que se están descomponiendo o arden paulatinamente- repartidas directamente o que afectan a los ayuntamientos de Sta. María del Páramo, Valdefuentes, Trobajo del Cerecedo, Ferral del Bernesga, etc., en la provincia de León. Si la solución propuesta en Sogama procedía de una idea de hace 20 años -la incineración- que no ha funcionado en la planta de Cerceda, ahora los ecologistas ponen como modelo la gestión que se está llevando en Cataluña con unas infraestructuras más pequeñas (lo contrario de lo que se teme como realidad para el Cerrato-Palencia), en las que las plantas de compostaje están dando mejores resultados. Al hacerlos más pequeños y cercanos a la gente, esta se conciencia y produce menos residuos en origen, se evitará el largo transporte de los residuos, se fomentará el consumo responsable, la recogida selectiva puerta a puerta y el reciclaje. Los ecologistas, en el mismo artículo, proponen que las autoridades impliquen a todos -ciudadanos, productores, consumidores, asociaciones, colectivos, estamentos, políticos- de manera que "el coste del tratamiento sea asumido por los que originan los residuos" obligando a los propios fabricantes a que utilizasen determinado tipo de plásticos que fuesen biodegradables o "fácilmente reciclables".

Este problema no es exclusivo de Italia o de España, y que no sirva como excusa para relajarnos, pero situaciones similares las podemos leer en los periódicos sobre Holanda, "Basura en el pienso"; Francia "Basura nuclear francesa rumbo a Rusia ya que desde 1990 buscan el sitio geológico ideal para depositar los residuos y esta búsqueda se prolongará hasta 2016" ¡26 años buscando el sitio ideal!, o Gran Bretaña "Compañías británicas exportan basura a China".

En una propuesta muy innovadora y atractiva económicamente, se encuentra la basura radiactiva holandesa (YIMBY - Yes in my back yard) que se está convirtiendo más en un complejo turístico que en un cementerio nuclear ya que las autoridades permiten visitas guiadas al centro para que la gente se familiarice con la "seguridad" de la actividad que allí se realiza. Su propuesta: educar a las personas sobre el fenómeno de la radioactividad y demostrar que es posible manejar de manera segura y responsable estos desechos.

Buenas propuestas para problemas complejos que tardarán en encontrar soluciones aptas para todas/todos, incluso para el medio ambiente. Y si realizamos la siguiente afirmación "Los residuos, desechos, basuras son un gasto que en la sociedad actual no nos podemos permitir, hecho que puede ser considerado un fracaso $\mathrm{y}$, cuando menos, un despilfarro" seguramente tendremos que realizar un análisis de la producción industrial que, 
por la diversidad de sectores, la dimensión de la empresa y su estructura organizativa, se pueden encontrar en una o varias de las fases de la fabricación.

\section{ANÁLISIS EN ORIGEN}

Para poder implantar un programa de prevención ambiental en origen es más fácil si se logran definir las características particulares de la empresa, es decir dibujar un mapa de la empresa y de los procesos de producción que nos ayudan a detectar lo superfluo e innecesario, las pérdidas, el despilfarro y, en definitiva, el fracaso de alguna de las fases conducentes a la elaboración de un producto, sea antes, durante y después. Lo analizaremos en los siguientes aspectos (Elías, 2009:55): Complejidad técnica de la fabricación, desde individualizada a grandes cantidades en un proceso de flujo continuo.

Si se fabrican productos diferentes, interesa conocer la distribución porcentual de los diversos productos y las secuencias de producción. Los problemas ambientales estarán concentrados en unos pocos productos o etapas de producción, que serán los que exigirán mayor atención.

Otro de los elementos fundamentales es la contabilidad ambiental. Pues producción ecoeficiente significa tener en cuenta tanto los aspectos ambientales como los económicos en el análisis y la toma de decisiones. Para demostrar los beneficios de la ecoeficiencia suele precisarse una contabilidad ambiental que permita conocer claramente los costes ambiéntales que tiene la empresa en el momento presente y el origen de los mismos. La contabilidad convencional clasifica los costes dentro de los siguientes grupos: Gastos directos; indirectos de fabricación; de ventas; generales y administrativos y los dedicados a investigación y desarrollo.

Un análisis en profundidad permite distinguir distintos tipos de costes que, de acuerdo con sus características y evidencia, pueden clasificarse como:

-Convencionales: son los más evidentes y fáciles de tener en cuenta. Debe recordarse el menor consumo de materias primas o menor coste que pueda haber en el tratamiento o disposición de las corrientes residuales.

-Potencialmente ocultos: incluyen aquellos gastos que se producen antes de iniciar la operación como el diseño previo de un mejor proceso, calificación de los suministradores, etc., y aquellos que no se han anticipado formalmente, pero que necesariamente se producirán en el futuro como el cierre y seguimiento de un vertedero.

-Contingentes: pueden producirse en el futuro y solamente se pueden valorar junto con un cierto grado de probabilidad.

-De imagen: destinados a afectar la percepción de clientes y público en general para los cuales es difícil conocer los beneficios directos que producirán.

La empresa, en su proceso de implantación de la gestión ambiental, puede que no lo perciba directamente, y en primer lugar, como un beneficio pues los cambios a introducir se suelen asimilar lentamente y cambiar la mentalidad de, casi, cada uno de los individuos que forman parte, directa o indirectamente, de todo el proceso de producción, pero también en otros aspectos importantes como puede ser el almacenaje o el transporte. Algunos resultados que podrían ser inmediatos: reducción en el consumo de agua y de otros recursos energéticos; disminuir la cantidad de residuos generados; disminuir la contaminación atmosférica y acústica; mejorar su imagen social y aumentar la capacidad competitiva de la empresa, que si bien ya no es determinante en positivo, sí lo sería en negativo si por cualquier hecho negativo sale a la luz que tal empresa no está adherida a este compromiso medioambiental. Entrar en 
esta dinámica representa el intento serio de que en nuestra empresa no se produzca despilfarro ni en la cantidad de materia prima utilizada inicialmente, ni en el residuo que nuestro producto genere después de su vida útil.

Figura 1

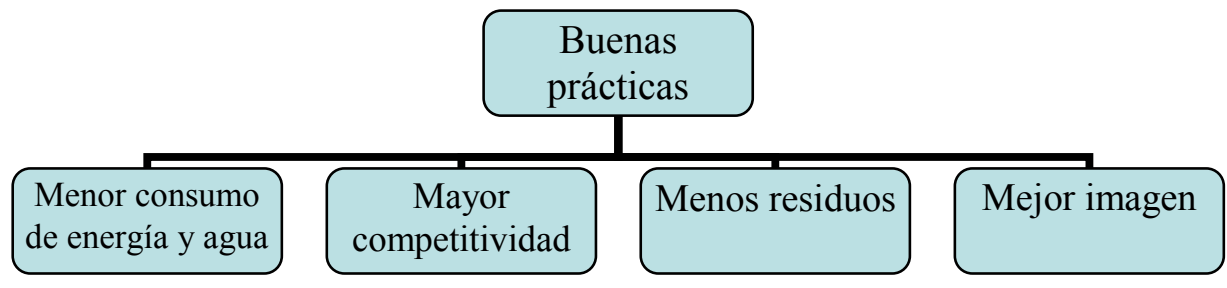

Fuente: Adaptación de Elías, 2009:62

Para concluir diremos que "No son basuras, sí materias primas" como puerta para introducirnos en el reciclaje.

¿En qué consiste el reciclaje? En principio, y según Seoánez Calvo (2000:56) el reciclaje está muy relacionado con la ecoeficiencia y destaca uno de los aspectos a entender bajo este concepto, más cercano a la reutilización o recuperación (sobre estas estadísticas hablaremos en otro momento) que al de reintroducción del desecho en el sistema de generación de un nuevo producto: Este tipo de economía, la ecoeficiencia, apunta hacia el desarrollo económico basado en el máximo aprovechamiento de los productos y de los servicios obtenidos a partir de: La utilización masiva de productos más duraderos; uso de los servicios y menos de los productos; medidas a favor de la producción no contaminante, economía energética, tecnologías limpias, reducción de residuos y reutilización; medidas de reciclado, recuperación, etc.

Para Seoánez Calvo, la reutilización de productos supone una reducción del flujo de materiales en el ciclo de la producción. El alargamiento del ciclo de vida de un producto tiene como consecuencia la sustitución de energía por mano de obra. La reparación de un producto moviliza mano de obra cualificada y tiene suficiente con un bajo consumo energético. Por lo tanto el recurso a los servicios de prolongación de vida de los productos, parece ofrecer ventajas no solamente desde el punto de vista del medio ambiente.

Para Elías, (2009:67-70), el concepto más general de reciclaje consiste en hallar el medio para sacar algún provecho del residuo que, como es natural, también tiene sus límites, tanto en el proceso de recogida (por ejemplo la logística necesaria para determinados residuos poco habituales o poco valiosos) como en la energía necesaria para su reintroducción en el proceso generador (por ejemplo en el caso del aluminio que de las $56.150 \mathrm{Kcal} . / \mathrm{Kg}$. necesarias en el proceso inicial, se pasa a $3.110 \mathrm{Kcal} . / \mathrm{Kg}$. en el proceso secundario o de reciclaje y eso conlleva un ahorro del $90 \%$, en el caso del papel o del vidrio supone un ahorro del $26 \%$ y del $28 \%$ respectivamente) así como ecológicos (por ejemplo la recogida de envases de latas de acero, cuya recuperación es realmente fácil pero el proceso de lavado, desestañado de las tapas, etc., genera más residuos que su depósito en un vertedero controlado pues es un residuo, metal, relativamente biodegradable y cuestiona el reciclado tanto ecológica como económicamente) y económicos (quizás el más fácil de 
delimitar al poder comprobar todos los costes que genera un residuo hasta que se vuelve a introducir en la cadena de consumo).

La valorización también produce un impacto en el medio ambiente que se debe minimizar. Por otro lado, y como hemos analizado anteriormente, los modelos de comportamiento y consumo de la sociedad se han de cambiar drásticamente para facilitar la valorización de los residuos pues sin el desarrollo de mercados no se puede cerrar el ciclo.

\section{CONCLUSIÓN}

Cuanto más tratemos de alejar de nuestra vista nuestros residuos más tardaremos en entender las consecuencias de nuestros actos para el medio ambiente, el otro medio ambiente, el que no queremos ver. Nuestro actuar perverso puede comenzar con el consumo, no el necesario para sobrevivir, como el que realizan miles de millones de personas sobre este planeta, sino el ostentoso. Podríamos considerar al individualismo ostentoso e incluso al turboconsumismo como lícito pero estas dos caras de la misma moneda suponen tal despilfarro para el medio ambiente que lo hacemos insostenible en el tiempo. Las señales de alarma hace tiempo que sonaron pero seguimos haciendo oídos sordos al tantán por el sonido de fondo contradictorio de los diferentes intereses tanto políticos como económicos. Por una parte no nos hacemos cargo de los residuos que generamos, los alejamos lo más posible de nuestro entorno y, por otra, el medio ambiente debe soportar nuestro detritus durante cientos o miles de años, como los CFC (aire), las bolsas de plástico, de moda hoy, los residuos radiactivos (suelos) o la contaminación de las aguas (aguas), etc. Contra los dos primeros se ha iniciado la batalla que, si bien fueron recibidos como prácticos y grandes descubrimientos, se han demostrado como muy dañinos. Supongamos por un momento que sus descubridores, los científicos, no lo sacan al mercado. Nos hubiesen librado de estas innecesarias plagas. Un tenue matiz positivo es la reacción de la sociedad, es un avance que usuarios, empresas y políticos se hayan dado cuenta y comenzado el movimiento, aunque no es suficiente. Nuestra responsabilidad, en este dilema, no es sólo por hechos acontecidos en el pasado, también por las consecuencias que puede tener para el futuro. No nos podemos permitir residuos para 400 o 4.000 años. Para ello y en el ámbito legislativo es necesaria la existencia de un único Plan de residuos, lo más globalmente posible, que promueva la reducción, segregación y reciclaje, socialmente aceptado, políticamente comprometido, asumido y consensuado, viable económicamente y medioambientalmente correcto.

Aquí nos quedamos. Seguramente para la próxima ocasión, hasta nosotros nos habremos reciclado o, quizás, hayamos sido valorizados, esperemos, en forma positiva.

\section{NOTAS}

El presente texto ha sido seleccionado de entre los presentados al XIV Congreso Nacional de Sociología en Castilla-La Mancha que, organizado por la Asociación Castellano-Manchega de Sociología, se celebró en Almagro (Ciudad Real), los días 13, 14 y 15 de noviembre. 


\section{BIBLIOGRAFÍA}

ALONSO, L. E. (2005): La era del consumo, Madrid, Siglo XXI.

ALTVATER, E. (1994): El precio del bienestar. Expolio del medio ambiente y nuevo (des)orden mundial, Valencia, Alfons el Magnànim.

BAUMAN, Z. (2007a): Tiempos líquidos, Barcelona, Tusquets.

- (2007b): Los retos de la educación en la modernidad líquida, Barcelona, Gedisa.

- (2007c): Vida de consumo, Madrid, FCE.

- (2000): Trabajo, consumo y nuevos pobres, Barcelona, Gedisa.

BECK, U. (2002): La sociedad del riesgo global, Madrid, Siglo XXI.

BOFF, L. (2008): La opción-Tierra, Santander, Sal Terrae.

BOORMAN, N. (2009): No marcas. Diario de un anticonsumista, Madrid, Temas de hoy.

BOURDIEU, P. (2003): Las estructuras sociales de la economía, Barcelona, Anagrama.

- (1988): La distinción. Criterio de bases sociales del gusto, Madrid, Taurus.

BRAUNGART, M y MCDONOUGH, W. (2005): Cradel to cradel. Rediseñando la forma en que hacemos las cosas, Madrid, McGraw-Hill.

CALVO VILlORIA, B. (2009): "Ramón Fernández Durán. El fin del petróleo", en Agenda Viva. Ciencia y Medioambiente en Madrid, No 16, Verano 2009, pp.12-18

DEAN, M. (2008): "Nápoles, donde la basura es oro", en http://www.jornada.unam.mx 07/01/2008.

DEL VAL, A. (1998): El libro del reciclaje, Barcelona, Integral.

DE SEBASTIÁN, L. (2009): Un planeta de gordos y hambrientos, Barcelona, Ariel.

DÍAZ CANO, E. y DÍAZ CANO, M. (2008): "Ecología en desequilibrio", en Pérez Redondo, R; García Manso, A. y Escribano Castellanos, M. (coords.) (2008): Sociedad, Consumo y Sostenibilidad, Toledo, Instituto de Consumo de Castilla-La Mancha y Asociación Castellano-Manchega de Sociología, pp. 249-262.

ELÍAS, X. (ed.) (2009): Reciclaje de residuos industriales, Madrid, Díaz de Santos.

Eurostat Statistical Books: Europe in Figures - Yearbook 2008, en la red:

http://epp.eurostat.ec.europa.eu/pls/portal/url/page/PGP_DS_YEARBOOK/PGE_DS_YEARBOOK_01

GALBRAITH, J. K. (2004): La economía del fraude inocente, Barcelona, Crítica.

- (1987): La sociedad opulenta, Barcelona, Ariel.

GARCÍA CALVO, A., et al. (1994): Sociología del consumo, Madrid, Edit. Complutense.

GIDDENS, A. (2001): Las nuevas reglas del método sociológico, Buenos Aires, Amorrortu.

GÓMEZ, M.V. (2009): “Todas las energías son necesarias”, en El País, 26/08/2009, pp.28-29.

GORZ, A. (2000): Miserias del presente, riqueza de lo posible, Buenos Aires, Paidós.

LATOUCHE, S. (2007): Sobrevivir al desarrollo, Barcelona, Icaria.

LIPOVETSKY, G. (2007): La felicidad paradójica. Ensayo sobre la sociedad de hiperconsumo, Barcelona, Anagrama.

- (2003): Metamorfosis de la cultura liberal, Barcelona, Anagrama.

LOVELOCK, J. E. (2007): La venganza de la tierra, Barcelona, Planeta.

LUCCHINI, L. (2008): "Nápoles pasa de la crisis de la basura a la emergencia sanitaria“, en El País, 12/01/2008.

LUHMANN, N. (1998): Sociología del riesgo, México, Universidad Iberoamericana.

MILLS, C.W. (1973): White-Collars. Las clases medias en Norteamérica, Madrid, Aguilar.

MORA, M. (2008): “Alemania halla restos radiactivos en la basura enviada desde Nápoles”, en El País, 07/06/2008.

NAPOLEONI, L. (2008): Economía canalla: la nueva realidad del capitalismo, Barcelona, Paidós.

NOGUEIRA, M. (2009): "Los ecologistas rechazan de plano ampliar o construir otra Sogama", en http://www.elcorreogallego.es, 28/05/2009.

RIFKIN, J. (2007): La economía del hidrógeno, Barcelona, Paidós.

RITZER, G. (1996): La McDonalización de la sociedad, Barcelona, Ariel.

ROMERO, D. (2009): "RE-CICLAR/des-ciclar. Lugares comunes en el lenguaje ecologista" en Agenda Viva. Ciencia y Medioambiente en Madrid, № 16, verano 2009, pp. 6-8. 
ROSENTHAL, E. (2009): "Hacia un nuevo tratado sobre el cambio climático. Más allá del Protocolo de Kyoto", en La Gaceta, 18/06/2009, p.24.

SARTORI, G. y MAZZOLENI, G. (2003): La tierra explotada, Madrid, Santillana.

SEOÁNEZ CALVO, M. (2000): Tratado de reciclado y recuperación de productos de los residuos, Madrid, Mundi-Prensa.

STIGLITZ, J.E. (2002): El malestar en la globalización, Barcelona, Círculo de lectores.

TAPSCOTT, D. y WILLIAMS, A.D. (2007): Wikinomics. La nueva economía de las multitudes inteligentes, Barcelona, Paidós.

TOFFLER, A. (1981): La tercera ola, Barcelona, Plaza\&Janés.

VAQUERO DÍAZ, I. (2004): Manual de diseño y construcción de vertederos de residuos sólidos urbanos, Madrid, E.T.S.I. Minas-U.P.M.

VEBLEN, T. (2002): Teoría de la clase ociosa, Madrid, FCE.

VERDÚ, V. (2005): Yo y tú, objetos de lujo, Barcelona, Debate.

WEIZSÄCKER, E.U.; LOVINS, L. H. y LOVINS, A. B. (1997): Factor 4, Barcelona, Círculo de Lectores.

\section{Breve currículo:}

\section{Manuel Díaz Cano}

Licenciado en Veterinaria por la Universidad de León. Integrante y responsable de los programas de erradicación de enfermedades animales en Asturias y Galicia. Jefe de Servicio de Sanidad Animal en Asturias y Galicia- Subdirector General de Sanidad Animal y Vegetal, Director General de producción Agropecuaria, Director del Instituto Lácteo y Ganadero y Director de Formación e Investigación Agraria, Jefe del Servicio de Gestión e Intervención Ambiental y de Programas y Gestión Ambiental y, actualmente, Subdirector General de Evaluación Ambiental, además de ponente en diferentes jornadas nacionales e internacionales sobre ganado vacuno, porcino y pesca o sobre estrategias para la erradicación de enfermedades de baja prevalencia.

\section{Eduardo Díaz Cano}

Profesor de Sociología y Medio Ambiente, en la Universidad Rey Juan Carlos e investigador de la Cátedra de Turismo de Madrid, realizó sus estudios en universidades de Alemania, Austria y Reino Unido, es Doctor en Sociología, recientemente ha publicado Una aproximación a Ernst Troeltsch (2009) y colaborado en diversos artículos y comunicaciones sobre Teoría sociológica, Medio ambiente y sociedad, Inmigración y Técnicas educativas así como en varios proyectos de investigación sobre la Educación y Cultura en la Unión Europea dentro de los Programas Sócrates / Leonardo y Comenius. 